\title{
Influence of heavy doping on Seebeck coefficient in silicon-on-insulator
}

\author{
H. Ikeda ${ }^{a)}$ and F. Salleh \\ Research Institute of Electronics, Shizuoka University, 3-5-1 Johoku, Naka-ku, Hamamatsu 432-8011, Japan
}

(Received 9 November 2009; accepted 10 December 2009; published online 6 January 2010)

\begin{abstract}
We measured the Seebeck coefficient of heavily P-doped silicon-on-insulator layers with $\mathrm{P}$ concentrations above $1 \times 10^{19} \mathrm{~cm}^{-3}$. The coefficient decreased with increasing P concentration, and with a peak of the Seebeck coefficient around $5 \times 10^{19} \mathrm{~cm}^{-3}$. We calculated the density-of-states (DOS) of bulk Si based on theoretical models of impurity-band formation, ionization-energy shift, and conduction-band tailing. The calculated impurity-concentration dependence of the energy derivative of the DOS at the Fermi energy also showed a peak. Consequently, the Seebeck coefficient of the heavily doped Si is ruled by the DOS distribution, similar to metallic materials. (C) 2010 American Institute of Physics. [doi:10.1063/1.3282783]
\end{abstract}

Thermoelectric devices have attracted considerable attention due to their ability to produce electric power from waste heat and as one means of tackling the global warming problem; however, the thermoelectric efficiency is still not sufficient for practical use. In order to enhance the efficiency, the figure-of-merit $Z$, which is proportional to the square of the Seebeck coefficient $S$ and the electric conductivity $\sigma$, and is inversely proportional to the thermal conductivity $\kappa$, has to be drastically increased. However, $S, \sigma$, and $\kappa$ strongly depend on the carrier concentration and are not independent of each other. Therefore, it is difficult to find the optimum condition of the material parameters. From a simple calculation, the optimum carrier concentration is considered to lie in the heavy-doping range of $10^{18}-10^{19} \mathrm{~cm}^{-3}$. ${ }^{1,2}$

We previously reported the carrier-concentration dependence of the Seebeck coefficient of P-doped ultrathin siliconon-insulator (SOI) layers and found that the Seebeck coefficient is enhanced at carrier concentrations above 3.5 $\times 10^{19} \mathrm{~cm}^{-3} \cdot 3,4$ In the present study, we investigate the Seebeck coefficient of heavily doped SOI layers and discuss the influence of a large number of impurity atoms. It will be demonstrated that the Seebeck-coefficient enhancement in the heavily doped samples is caused by the influence of impurity-band formation, ionization-energy shift, and conduction-band tailing.

The SOI wafer used consisted of a top Si layer (SOI layer), a $400 \mathrm{~nm}$ thick buried oxide (BOX) layer and a $p$-type $\mathrm{Si}$ substrate. The SOI layer was thinned to a thickness of 6-100 $\mathrm{nm}$ by oxidizing the SOI surface and removing the formed oxide. ${ }^{3,4} \mathrm{P}$ atoms were doped into the SOI layer by thermal diffusion to form an $n$-type Si layer. The carrier concentration ranged from $1 \times 10^{18}$ to $1 \times 10^{20} \mathrm{~cm}^{-3}$, as determined by a four-probe method at room temperature. The time evolution of the thermoelectromotive force was measured, simultaneous with temperatures, at the high- and lowtemperature regions in the sample. The Seebeck coefficient was evaluated from the thermoelectromotive force $(\Delta V$ $\left.\equiv V_{H}-V_{L}\right)$ and the temperature difference $\left(\Delta T \equiv T_{H}-T_{L}\right)$ by $S=\Delta V / \Delta T$.

The absolute value of the measured Seebeck coefficient of the SOI layers is shown in Fig. 1 as a function of carrier concentration. The numbers adjacent to the filled circles in-

${ }^{a)}$ Electronic mail: ikeda@rie.shizuoka.ac.jp. dicate the SOI layer thickness. In this figure, the results for $n$-type Si wafers obtained from our measurements and reported in the literature ${ }^{5-7}$ are also shown. The solid line is the theoretical Seebeck coefficient obtained from our calculations based on the dispersion relation in simple bulk $\mathrm{Si}^{8}{ }^{89} \mathrm{As}$ mentioned in our previous letters, ${ }^{3,4}$ the values of the Seebeck coefficient for SOI layers appear to be very similar to those for bulk Si wafers lying on a curved line (indicated by the broken line in Fig. 1) at carrier concentrations below $3.5 \times 10^{19} \mathrm{~cm}^{-3}$. This means that the measured Seebeck coefficient hardly includes the influence of the $p$-type Si substrate. The difference between experimental and theoretical results is considered to be due to the influence of phonon drag. ${ }^{3,4,6}$ In this carrier-concentration range, the absolute value of the experimental Seebeck coefficient of the SOI layers decreases with an increase in the carrier concentration, as is generally seen in semiconductor materials. ${ }^{1}$

On the other hand, SOI layers with carrier concentrations above $3.5 \times 10^{19} \mathrm{~cm}^{-3}$ are found to exhibit unusual behavior in their Seebeck coefficients, as shown by the dotted line in Fig. 1. It is clearly seen that the absolute value of the Seebeck coefficient increases with increasing carrier concentration, and then decreases again at $1.6 \times 10^{20} \mathrm{~cm}^{-3}$. The 6.7 and $67.2 \mathrm{~nm}$ thick SOI samples exhibit the enhanced Seebeck coefficient, which suggests the SOI thickness above $6 \mathrm{~nm}$ does not contribute to the enhancement in the Seebeck

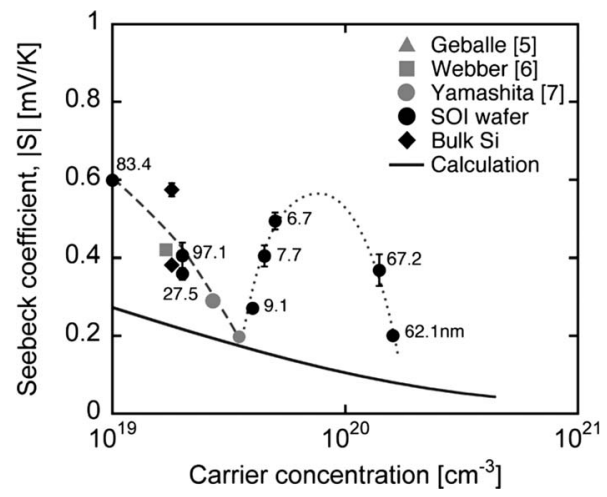

FIG. 1. Absolute Seebeck coefficient of SOI wafers as a function of carrier concentration. The Seebeck coefficients of Si wafers obtained from our measurements and reported in the literature (Refs. 5-7) are also shown. The solid line represents the calculated value, and the broken and the dotted lines are drawn as an eye-guide. 


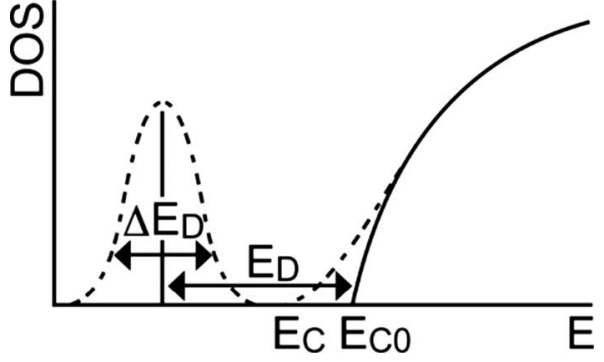

FIG. 2. Schematic of DOS distribution in an impurity level and a conduction band for a heavily doped semiconductor. $E_{\mathrm{D}}$ and $\Delta E_{\mathrm{D}}$ are the ionization energy and the energy width of the impurity band, respectively. $E_{\mathrm{C} 0}$ and $E_{\mathrm{C}}$ represent the original and the shifted conduction-band edges, respectively.

coefficient. Therefore, the result of Fig. 1 is likely due to the influence of the formation of an impurity band. ${ }^{10}$

We consider three principal influences of heavy doping in an $n$-type semiconductor. The first is the formation of an impurity band originating from the overlap between wave functions of bound electrons. ${ }^{11,12}$ With increasing impurity concentration, the bandwidth $\Delta E_{\mathrm{D}}$ expands and the density of states (DOS) becomes broad from a $\delta$-function state. The second is the ionization-energy $E_{\mathrm{D}}$ shift of an impurity atom due to the screening effect by conduction electrons. ${ }^{13,14}$ The presence of the conduction electrons should screen the attractive interaction between the donor ion and the bound electron in a donor level. This screening will shift the energy of the ground state toward the conduction band. The third is band tailing at the conduction band edges owing to disorder in the atomic arrangement. The variation of the local electrostatic potential in the lattice causes fluctuations in energy in electronic states in the conduction band and thus produces a tail on the conduction-band DOS. ${ }^{13-16}$ These influences are schematically depicted in Fig. 2.

The energy width of an impurity band was calculated for P-doped bulk Si, based on a Baltensperger model. ${ }^{11}$ This model assumes a regular close-packed lattice built of hydrogenlike impurities, where the Schrödinger equation is solved inside a sphere occupied by an impurity atom. The $\Delta E_{\mathrm{D}}$ is defined as the energy difference between the band edges obtained from boundary conditions for the wave functions. The wave function includes the confluent hypergeometric series $F\left(l+1-n, 2 l+2 ; 2 r_{s} / n a_{\mathrm{B}}\right)$ in the hydrogenlike model, ${ }^{11}$ where $a_{\mathrm{B}}$ is the Bohr radius and $r_{s}$ is the mean radius of the sphere occupied by an impurity atom. The indices $n$ and $l$ are the principal and the orbital quantum numbers, respectively. In this letter, the impurity band width is computed under the condition that the principal quantum number $n$ is approximately equal to unity for $l=0$, corresponding to a $1 \mathrm{~s}$ band. ${ }^{17}$ After obtaining the band width, a Gaussian curve is used to represent the actual DOS across the impurity band, with the maximum lying at the energy level corresponding to the original impurity level. ${ }^{12} \mathrm{We}$ can express the impurity concentration as $N_{\mathrm{D}}=\Delta E_{\mathrm{D}} N_{\mathrm{D}} / 2 \sqrt{2 \pi} \sigma_{i}$, where $\sigma_{i}$ is a parameter characterizing the extent of the Gaussian function. This expression assumes that the Gaussian function has a triangular shape with a base of $\Delta E_{\mathrm{D}}$ and a height of $N_{\mathrm{D}} / \sqrt{2 \pi} \sigma_{i}$. The DOS function for the impurity band can then be represented by

$$
\rho_{i}(E)=\frac{2 N_{\mathrm{D}}}{\Delta E_{\mathrm{D}}} \exp \left\{-4 \pi\left(\frac{E-E_{\mathrm{D}}}{\Delta E_{\mathrm{D}}}\right)^{2}\right\},
$$

where the conduction band edge $E_{\mathrm{C}}$ is set to zero.

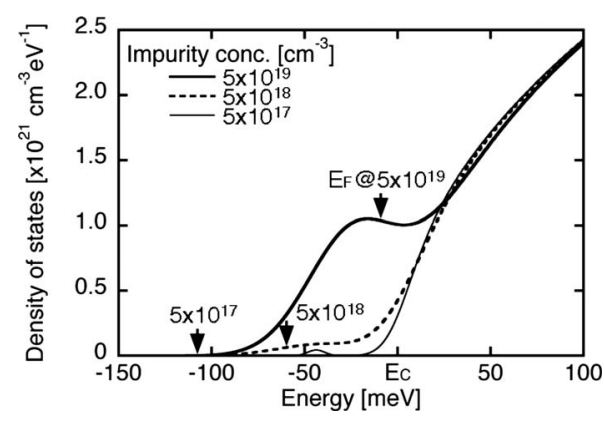

FIG. 3. Density of states calculated for an impurity concentration of $N_{\mathrm{D}}$ $=5 \times 10^{17}, 5 \times 10^{18}$, and $5 \times 10^{19} \mathrm{~cm}^{-3}$, based on the three influences of the heavy doping. The original ionization energy is set to $E_{\mathrm{D}}=-44 \mathrm{meV}$ for the $\mathrm{P}$ atom and the arrows indicate the Fermi energies.

The ionization energy $E_{\mathrm{D}}$ and the conduction-band edge tailing were theoretically formularized in literatures. ${ }^{13,14}$ The first-order perturbation theory of the potential difference between the screened and unscreened ions can be used to estimate the ionization-energy shift. The calculated result for the shift is

$$
\delta E_{i}=\frac{q^{2} \xi}{4 \pi \epsilon}\left\{\frac{3}{8}-\frac{2 \xi \lambda_{e} \sin \left[\left(1 / 2 \xi \lambda_{e}\right)+2 \tan ^{-1}\left(1 / 8 \xi \lambda_{e}\right)\right]}{4+1 / 16 \xi^{2} \lambda_{e}^{2}}\right\},
$$

where $q$ is the elemental charge, $\epsilon$ the dielectric constant, and $\lambda_{e}$ the screening length. $\xi$ is the reciprocal of the Bohr radius for the donor $\left(\xi=1 / a_{\mathrm{B}}\right)$.

With regards to the conduction-band tailing, the average conduction-band DOS can be represented by integrating the product of the local DOS at a point with potential $V$ and the distribution of the potential. Assuming that the local DOS $\rho_{\mathrm{C}}(E)$ is for slowly varying potential fluctuations and that the distribution potential $p(V)$ is approximately Gaussian, ${ }^{15,16}$ the conduction-band DOS is given by ${ }^{13}$

$$
\begin{aligned}
N_{\mathrm{C}}(E)= & \int_{-\infty}^{E} \rho_{\mathrm{C}}(E-V) \times p(V) d V=\int_{-\infty}^{E} \frac{6 \sqrt{2}\left(m^{*}\right)^{3 / 2}}{\pi^{2} \hbar^{3}} \sqrt{E-V} \\
& \times \frac{1}{\sqrt{2 \pi} \sigma_{\mathrm{C}}} \exp \left(-\frac{V^{2}}{2 \sigma_{\mathrm{C}}^{2}}\right) d V,
\end{aligned}
$$

where $m^{*}$ is the effective mass and $\hbar$ is Plank's constant. The standard deviation is given by $\sigma_{\mathrm{C}}=\sqrt{N_{\mathrm{D}} q^{4} \lambda_{e} / 8 \pi^{2} \epsilon^{2}}$. Note that the mobility of electrons in the DOS tail is a function of the energy, approaching zero for energies below a certain level in the tail. In this calculation, for simplicity, the mobility is assumed to be constant for energies greater than $-2 \sigma$ and zero for energies less than $-2 \sigma$.

The calculated resultant DOS, taking into account the influence of the heavy doping mentioned above, is shown in Fig. 3 for impurity concentrations of $5 \times 10^{17}, 5 \times 10^{18}$, and $5 \times 10^{19} \mathrm{~cm}^{-3}$. The original ionization energy is set to $E_{\mathrm{D}}=-44 \mathrm{meV}$ for $\mathrm{P}$ atoms in $\mathrm{Si}$. The arrows in this figure indicate the positions of the Fermi energy evaluated from the charge-neutrality condition. It is found that for 5 $\times 10^{17} \mathrm{~cm}^{-3}$ the DOS of the impurity band is small and away from the conduction band. In addition, the Fermi energy stands in the band gap and is far from the conduction band. These facts mean that the SOI layer still has the characteristics of a semiconductor. In the cases of $5 \times 10^{18}$ and $5 \times 10^{19} \mathrm{~cm}^{-3}$, on the other hand, the impurity band is con- 


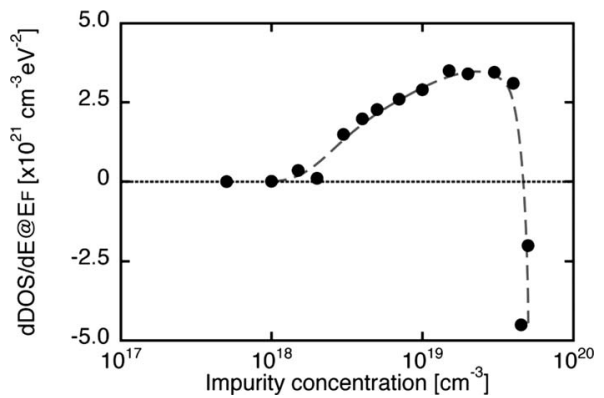

FIG. 4. Energy derivative of DOS at the Fermi energy, computed on the basis of the three influences of the heavy doping, as a function of impurity concentration. The broken line is drawn as an eye-guide.

nected with the conduction band, and the Fermi energy stays in the continuous band. This band arrangement should exhibit metallic properties.

According to the Mott equation, the Seebeck coefficient is proportional to the energy derivative of the DOS at the Fermi energy $\left(d N\left(E_{\mathrm{F}}\right) / d E\right)$. ${ }^{18}$ This theory is considered to be valid for metallic materials. We computed the $d N\left(E_{\mathrm{F}}\right) / d E$ for heavily doped Si from the calculated DOS. Figure 4 shows the computed results as a function of impurity concentration. It is clearly found that the $d N\left(E_{\mathrm{F}}\right) / d E$ increases with increasing impurity concentration above $1 \times 10^{18} \mathrm{~cm}^{-3}$ and abruptly decreases around $5 \times 10^{19} \mathrm{~cm}^{-3}$; that is, there is a peak around $1 \times 10^{19} \mathrm{~cm}^{-3}$. This result is qualitatively in good agreement with the experimental result of Fig. 1 although the horizontal axis shifts by an order of magnitude. The quantitative reproducibility strongly depends on the physical model. Hence, the difference in the impurity (carrier) concentration axis between Figs. 1 and 4 is probably due to lack of quantitative validity in the models of impurity-band DOS, ionization-energy shift and conduction-band tailing. Note that the solid line in Fig. 1 is calculated on the basis of the dispersion relation in simple bulk Si. At $3.5 \times 10^{19} \mathrm{~cm}^{-3}$, the experimental Seebeck coefficient is nearly equal to the theoretical value since the phonon-drag influence is not considered to be significant in this carrier-concentration range. Therefore, it is likely that the SOI layer becomes metallic above $3.5 \times 10^{19} \mathrm{~cm}^{-3}$. As a result, the metallic SOI layer has a peak in its Seebeck coefficient because the Seebeck coefficient of metallic materials is proportional to the $d N\left(E_{\mathrm{F}}\right) / d E$. $^{18}$

We have thus investigated the Seebeck coefficient of heavily-doped SOI layers with carrier concentrations of 1 $\times 10^{18}-2 \times 10^{20} \mathrm{~cm}^{-3}$ and found that the Seebeck coefficient has a peak around $3 \times 10^{19}-2 \times 10^{20} \mathrm{~cm}^{-3}$. By calculating the DOS distribution including the influence of impurity-band formation, ionization-energy shift, and conduction-band tailing, the $d N\left(E_{\mathrm{F}}\right) / d E$ also has a peak. These facts indicate that SOI layers above $3 \times 10^{19} \mathrm{~cm}^{-3}$ have metallic DOS and that the Seebeck coefficient is mainly ruled by the DOS distribution.

We would like to express our thanks to Professor A. Ishida for his support of the Seebeck coefficient calculation. This work was financially supported by a Grant-in-Aid for Scientific Research (Grant No. 21360336) from the JSPS.

${ }^{1}$ G. D. Mahan, Solid State Phys. 51, 81 (1997).

${ }^{2}$ D. M. Rowe, Thermoelectrics Handbook, Macro to Nano (CRC, Taylor \& Francis Group, Boca Raton, 2006), Chap. 1, p. 7.

${ }^{3}$ F. Salleh, K. Asai, A. Ishida, and H. Ikeda, Appl. Phys. Express 2, 071203 (2009).

${ }^{4}$ F. Salleh, K. Asai, A. Ishida, H. Ikeda, and J. Automation, J. Autom. Mobile Rob. Intell. Syst. 3, 49 (2009).

${ }^{5}$ T. H. Geballe and G. W. Hull, Phys. Rev. 98, 940 (1955).

${ }^{6}$ L. Weber and E. Gmelin, Appl. Phys. A: Mater. Sci. Process. 53, 136 (1991)

${ }^{7}$ O. Yamashita, J. Appl. Phys. 95, 178 (2004).

${ }^{8}$ A. Ishida, D. Cao, S. Morioka, Y. Inoue, and T. Kita, J. Electron. Mater. 38, 940 (2009).

${ }^{9}$ A. Ishida, T. Yamada, D. Cao, Y. Inoue, M. Veis, and T. Kita, J. Appl. Phys. 106, 023718 (2009).

${ }^{10}$ J. P. Heremans, V. Jovovic, E. S. Toberer, A. Saramat, K. Kurosaki, A. Charoenphakdee, S. Yamanaka, and G. J. Snyder, Science 321, 554 (2008).

${ }^{11}$ W. Baltensperger, Philos. Mag. 44, 1355 (1953).

${ }^{12}$ T. P. Brody, J. Appl. Phys. 33, 100 (1962).

${ }^{13}$ T. F. Lee and T. C. McGill, J. Appl. Phys. 46, 373 (1975).

${ }^{14}$ D. S. Lee and J. G. Fossum, IEEE Trans. Electron Devices 30, 626 (1983).

${ }^{15}$ E. O. Kane, Phys. Rev. 131, 79 (1963).

${ }^{16}$ T. N. Morgan, Phys. Rev. 139, A343 (1965).

${ }^{17}$ C. Erginsoy, Phys. Rev. 88, 893 (1952).

${ }^{18}$ N. F. Mott and E. A. Davis, Electronic Processes in Non-Crystalline Materials (Clarendon, Oxford, 1979), p. 52. 